\title{
Formation of nanoclusters under radiation pressure in solution: A Brownian dynamics simulation study
}

\author{
Prasanth P. Jose and Biman Bagchi ${ }^{\mathrm{a})}$ \\ Solid State and Structural Chemistry Unit, Indian Institute of Science, Bangalore-12, India
}

\begin{abstract}
When radiation is scattered by a medium, a part of its momentum is transferred to the target particles. This is purely a mechanical force which comes into effect when radiation is not coherently interacting. This force is known in literature as radiation pressure. Recent experimental studies have demonstrated the feasibility of using radiation pressure of a laser beam as a tool for cluster formation in solution. In this paper we describe the Brownian dynamics simulation of solute molecules under the perturbation induced by laser radiation. Here the force field generated by a laser beam in the fundamental mode is modeled as that of a two-dimensional harmonic oscillator. The radial distribution function of the perturbed system gives indication of high inhomogeneities in the solute distribution. An explicit analysis of the nature of these clusters is carried out by calculating the density-density correlation functions in the plane perpendicular to beam direction $g\left(r^{x y}\right)$; and along the direction of beam $g(z)$, they give an average picture of shell structure formation in the different directions. The relaxation time of the first shell structure calculated from the van Hove correlation function is found to be relatively large in the perturbed solution. This is the signature of formation of stable nanoclusters in the presence of the radiation field. Our study on the dynamics of solute molecules during the cluster formation and dissolution gives the duration of collective relaxation, far away from the equilibrium to an equilibrium distribution. This relaxation time is found to be large for a perturbed solution.
\end{abstract}

\section{INTRODUCTION}

Study of cluster formation in the solution is a subject of great current interest. ${ }^{1,2}$ Often the route used is a chemical reaction, followed by aggregation. ${ }^{3}$ Recently, however, an interesting technique has been developed where radiation pressure is used to selectively bring together specific particles and form a cluster. The merit of this technique is that clusters of desired shape can be formed which gives this technique a special advantage. In this work, we present a Brownian dynamics simulation of such a cluster formation in a uniform solution. This study reveals microscopic aspects of such a cluster formation.

Interaction of electromagnetic radiation with atoms and molecules via absorption and emission gives insight into the structure and dynamics of inner degrees of freedom. If the incoming radiation is not resonantly interacting, this interaction gives rise to a pure mechanical pressure which could be independent of the internal structure of atoms and molecules. The radiation pressure is the mechanical pressure exerted by radiation due to the partial transfer of the momentum of radiation while it is reflected or refracted. Alternatively, radiation pressure can be defined microscopically as the force experienced by a particle, when the dipole induced by external field on the particle tries to minimize its energy by repositioning. Debye in 1909 carried out a complete study of radiation pressure on spherical particles of arbitrary size and optical constants. ${ }^{4,5}$ The most general derivation of the magnitude of force generated by the radiation can be done by the

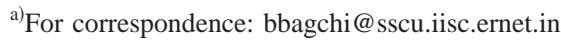

principle of conservation of momentum of incident radiation. ${ }^{5,6}$ There are two kinds of forces arising from the scattering of radiation. One is along the direction of propagation of radiation and the second one gives the transverse component, which is due to the scattering of a beam of radiation with an asymmetric distribution of intensity by the target particle. This creates a net force on the particle due to the difference in the momentum transferred at different parts of the target particle. The first experimental evidence of radiation pressure using sophisticated experiments after the advent of the lasers can be found in the celebrated work of Ashkin, where he reported acceleration of a single microparticle by radiation pressure. His successive works confirmed the effect of radiation pressure on huge biomolecules, latex particles, etc. ${ }^{7}$

This work pursues a different aspect of the problem, namely the statistical behavior of a collection of interacting molecules under the stable external force field created by a laser beam. Recently Masuhara et al. ${ }^{8-11}$ carried out extensive experimental work on microparticle formation in the solution by a radiation field. They also reported an analysis of the potential arising from the radiation which is found to be harmonic in nature. These experiments look into the classical many-body problem of cluster formation under an inhomogeneous force field. Another interesting aspect of the problem is the dynamics of formation of such clusters under the radiation field and dissolution of them when radiation is switched off.

The organization of the rest of the paper is as follows: Section II gives the details of modeling of radiation pressure 
as a harmonic potential. The details of the Brownian dynamics simulation are given in Sec. III. Results obtained from the simulation are described in Sec. IV. Some concluding remarks are presented in Sec. V.

\section{THEORETICAL FORMULATION}

Here we are interested particularly in the incoherent interaction of the molecules with radiation. For a coherent beam of radiation having Gaussian intensity distribution in the plane of cross-section the force field experienced by a molecule can be modeled analogous to that of a twodimensional harmonic oscillator with force constant $k$. When the electromagnetic radiation interacts with a dipole it experiences a Lorentz force ${ }^{12}$

$$
\mathbf{F}=(\mathbf{p} \cdot \nabla) \mathbf{E}+\frac{1}{c} \frac{\partial \mathbf{p}}{\partial t} \times \mathbf{E},
$$

where $\mathbf{E}$ is the electric field in the plane of beam crosssection and $\mathbf{p}$ is the dipole moment. This expression can be arranged using the vector identity $\mathbf{E} \cdot \nabla \mathbf{E}=\frac{1}{2} \nabla E^{2}-\mathbf{E} \times(\nabla$ $\times \mathbf{E})$ and $\mathbf{p}=\alpha \mathbf{E}, \quad \alpha$ is assumed to be the positive polarizability $^{12}$

$$
\mathbf{F}=\alpha\left(\frac{1}{2} \nabla E^{2}+\frac{1}{c} \frac{\partial(\mathbf{E} \times \mathbf{B})}{\partial t}\right) .
$$

Due to the heavy mass of the scatterer, the force in the direction of beam can be neglected, hence the total force can be approximated by first term of Eq. (2)

$$
\mathbf{F} \simeq \alpha \frac{1}{2} \nabla E^{2} .
$$

It is evident from this equation that the force due to radiation depends on the gradient of the magnitude of electric field in the transverse direction of the beam. Here we are assuming the laser beams is in the $\mathrm{TEM}_{00}$ mode. ${ }^{13}$ Hence the intensity distribution function is a Gaussian in the $x-y$ plane with the direction of beam propagation along the $z$ direction. Intensity of the beam as experienced by the $j$ th molecule is

$$
u_{j}=u_{0} \exp \left(\frac{-2 \mathbf{r}_{0 j}^{2}}{w_{0}^{2}}\right),
$$

where $r_{0 j}^{2}=x_{j}^{2}+y_{j}^{2}, \quad r_{0 j}$ is the displacement of $j$ th molecule from the radiation axis in the $x-y$ plane. $w_{0}$ is the spot size which is much larger than $r_{0 j} . u_{0}$ is the intensity at the center of the beam. Then the corresponding magnitude of electric field is described by $E=E_{0} \exp \left(-r_{0 j}^{2} / w_{0}^{2}\right)$. The force experienced on the $j$ th molecule by this stable potential well created by this laser beam can be derived by substituting this relation in Eq. (3)

$$
\mathbf{F}_{j}(r)=-\alpha E_{0}^{2} \frac{2 \mathbf{r}_{0 j}}{w_{0}^{2}} \exp \left(\frac{-2 \mathbf{r}_{0 j}^{2}}{w_{0}^{2}}\right) .
$$

Since $w_{j 0} \gg\left|\mathbf{r}_{0 j}\right|$ the Gaussian can be expanded in powers of $2 \mathbf{r}_{0 j}^{2} / w_{0}^{2}$,

$$
\mathbf{F}_{j}(r)=-\alpha E_{0}^{2} \frac{2 \mathbf{r}_{0 j}}{w_{0}^{2}}\left(1-\frac{2 \mathbf{r}_{0 j}^{2}}{w_{0}^{2}} \cdots\right),
$$

with in zeroth order approximation it can be written as

$$
\mathbf{F}_{j}(r) \simeq-2 \alpha E_{0}^{2} \frac{\mathbf{r}_{0 j}}{w_{0}^{2}},
$$

or

$$
\mathbf{F}_{j}(r) \simeq-k \mathbf{r}_{0 j},
$$

where

$$
k=\frac{2 \alpha E_{0}^{2}}{w_{0}^{2}} .
$$

Hence the force due to the radiation pressure of a laser beam in the fundamental mode can be approximated by a harmonic potential. The magnitude of the electric field can be calculated from the power of the laser. ${ }^{13}$

\section{SYSTEM AND SIMULATION DETAILS}

In the model simulated here, the solute molecules move in the solution under the force field generated by the sum of mutual interactions and the external field generated by the radiation pressure. Hence the motion of the solvent is analogous to that of a Brownian particle which moves under the random forces in a potential well. Hence a Brownian dynamics simulation, ${ }^{14,15}$ is the most suitable method for tracing the dynamics of solute molecules in the solution. In the Brownian dynamics simulation the solute molecules are selectively simulated as moving under the friction or resistance generated by solvent molecules. In our simulation the equation of motion is obtained by integrating the single particle Langevin equation, hence all the hydrodynamic effects are neglected. Simulation is confined to the particle rays of the laser beam since the difference in the angle between any two rays falling on a different part of a single molecule is negligible. In addition to this the entire simulation box occupies a small volume in comparison with the region of cluster formation in experimental arrangements used, ${ }^{8-11}$ hence the approximation used here is quite justifiable.

Other details of the simulation are as follows: A system consists of 500 molecules are selected for simulation. The simulation is carried out inside a cubical box (the schematic diagram of the simulation box is given in Fig. 1); this box is placed in the positive quadrant of the coordinate system. Hence the position coordinates along the $x, y$, and $z$ axis vary from zero to $l$, where $l$ is the length of the simulation box. In this simulation the box length is computed from the density of solute molecules and it is approximately $14 \sigma$ ( $\sigma$ is the molecular diameter) in this simulation. Intermolecular interaction is modeled through a Lennard-Jones potential with a cutoff at $r_{i j}=2.5 \sigma$. The intermolecular interactions is given by

$$
v_{i j}=4 \epsilon\left(\frac{\sigma}{r_{i j}^{12}}-\frac{\sigma}{r_{i j}^{6}}\right) .
$$

Hence total potential energy $V_{i}$ on the $i$ th molecule due to the intermolecular interaction is given by

$$
V_{i}=\sum_{j} v_{i j}
$$




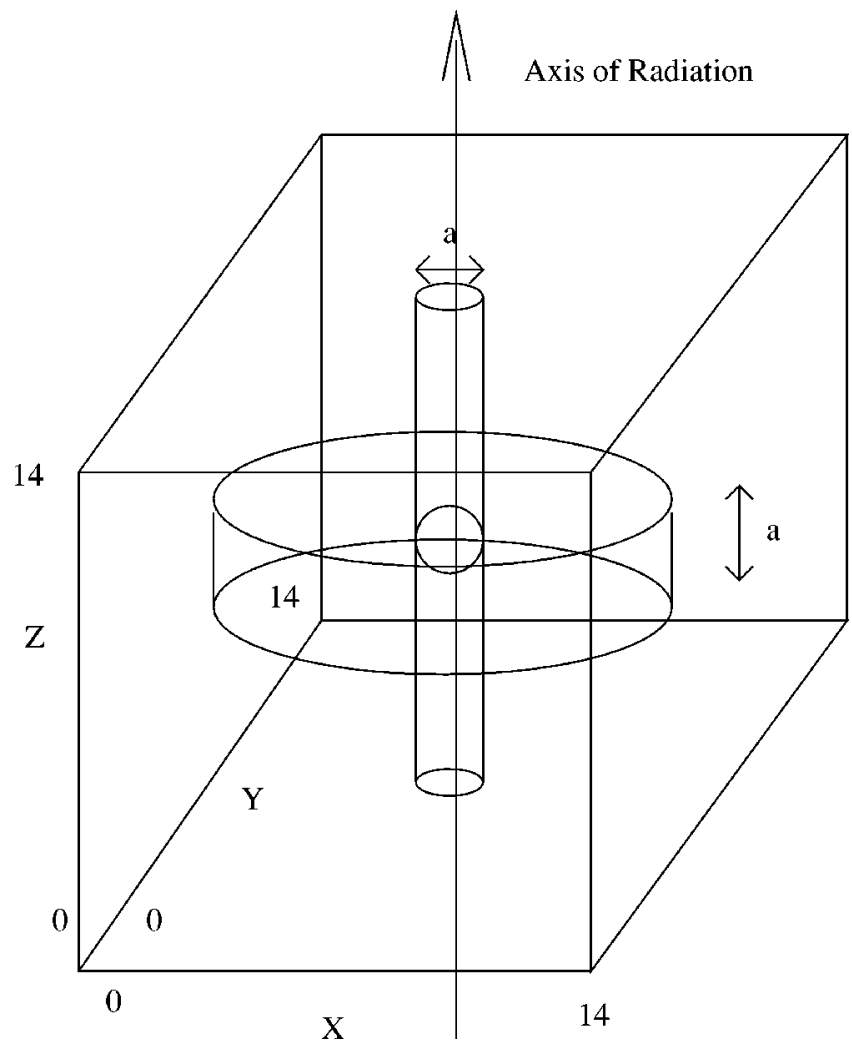

FIG. 1. The schematic diagram of the simulation box is shown in the figure. The disk and the cylinder used for calculating $g\left(r^{x y}\right)$ and $g\left(r_{i j}^{z}\right)$ are schematically drawn here. The thickness of the disk and the diameter of the cylinder are same $(a=\sigma)$.

Here radiation is passing symmetrically through the center of the $x-y$ plane of the simulation box and its direction of propagation is along the $z$ axis. Therefore the Gaussian intensity distribution of radiation is situated symmetrically around the center of the $x-y$ plane. The radiation force from this Gaussian beam is modeled as generated by a twodimensional harmonic potential and it can be written as

$$
V_{j}^{\mathrm{rad}}=\frac{1}{2} k r_{0 j}^{2} \text {. }
$$

The total potential energy is given by

$$
V_{\text {total }}=\sum_{j} V_{j}+V_{j}^{\mathrm{rad}} .
$$

By integrating the single particle Langevin equation, equation of motion is obtained as

$$
\mathbf{r}(t+\Delta t)=\mathbf{r}(t)+\frac{D \mathbf{f} \Delta t}{k_{B} T}+\mathbf{R}(\Delta t),
$$

where $\mathbf{f}$ is the total deterministic force, $D$ is the diffusion constant, and $k_{B}$ is the Boltzmann constant. The random displacements coming from the solvent molecules as sampled from Gaussian distribution whose mean is zero and variance is $\sqrt{2 D t}$ in all three directions $(x, y$, and $z$ ). The force constant $k$ is expressed as a dimensionless quantity as $k^{*}$ $=k \sigma^{2} / \epsilon$ and its value used in this simulation is 80.0. All other quantities used in the simulation are in reduced units using Lennard-Jones parameters. They are density $\rho^{*}=\rho \sigma^{3}$, temperature $T^{*}=k_{B} T / \epsilon$, distance $r^{*}=r / \sigma$ and time $t^{*}$ $=\sqrt{\epsilon / m \sigma^{2} t}$. The simulation is performed at a high concentration of the solute molecules at $\rho^{*}=0.2$ and at $T^{*}=1.2$. Here this external potential has the symmetry of a cylinder. That is, all the molecules situated on the curved surface of a cylinder, which is centered at the radiation axis, will experience a force equal in magnitude toward the center. Hence the bins which are used for recording the density are concentric cylindrical shells centered at the radiation axis. These shells are constructed such that all of them have equal volume.

The simulation starts from an fcc configuration and it is equilibrated with 20000 simulation steps. Periodic boundary condition is used in all the three directions. The equation of motion is integrated with the time step $t^{*}=0.005$. Positions of solute molecules are recorded (without perturbation) in the next $10^{5}$ steps, from this the radial distribution function $[g(r)]$ is calculated, which can be defined in terms of delta function as ${ }^{16}$

$$
g(\mathbf{r})=\frac{1}{N \rho}\left\langle\sum \sum_{i \neq j}^{N} \delta\left(\mathbf{r}+\mathbf{r}_{j}-\mathbf{r}_{i}\right)\right\rangle .
$$

$g(\mathbf{r})=g(r)$ in a homogeneous system. The conventional radial distribution function gives the distribution of the molecules in a homogeneous solution. Since the application of radiation pressure create a strong inhomogeneity in the solution, here the conventional $g(r)$ cannot say much about the distribution of molecules on $x-y$ plane and in the $z$ directions explicitly. To overcome this difficulty we have calculated the density-density correlation function in the $x-y$ plane, which can be defined in terms of delta function as

$$
g\left(\mathbf{r}^{x y}\right)=\left.\frac{1}{N \rho}\left\langle\sum \sum_{i \neq j}^{N} \delta\left(\mathbf{r}^{x y}+\mathbf{r}_{j}^{x y}-\mathbf{r}_{i}^{x y}\right)\right\rangle\right|_{|\Delta \mathbf{z}|<0.5 \sigma},
$$

where $\mathbf{r}^{x y}=\mathbf{r}-\mathbf{z}$ (or $\mathbf{r}^{x y}$ is the projection of displacement in the $x-y$ plane and $\Delta \mathbf{z}$ is the projection of displacement between two molecules along the $z$ axis). In the homogeneous solution the distribution function is related to the $g(r)$ by the relation $\lim _{|\Delta \mathbf{z}| \rightarrow 0} g\left(r^{x y}\right)=g(r)$, since all the directions are equivalent in a homogeneous solution. Similarly a distribution function along the $z$ axis can be defined as

$$
g(\mathbf{z})=\left.\frac{1}{N \rho}\left\langle\sum \sum_{i \neq j}^{N} \delta\left(\mathbf{z}+\mathbf{z}_{j}-\mathbf{z}_{i}\right)\right\rangle\right|_{\mid \Delta \mathbf{r}^{x y \mid}<0.5 \sigma},
$$

where $\mathbf{z}$ is the projection of displacement along the $z$ axis and $\Delta \mathbf{r}^{x y}$ is the projection of displacement between two molecules in the $x-y$ plane. Similar to the previous case $g(z)$ also related to the conventional $g(r)$ by the relation $\lim _{\left|\Delta \mathbf{r}^{x y}\right| \rightarrow 0} g(z)=g(r)$. For the calculation of the $g\left(\mathbf{r}^{x y}\right)$ and $g(\mathbf{z})$ we have chosen the limits $|\Delta \mathbf{z}|<0.5 \sigma$ and $\left|\Delta \mathbf{r}^{x y}\right|$ $<0.5 \sigma$, respectively, rather than the $|\Delta \mathbf{z}| \rightarrow 0$ and $\left|\Delta \mathbf{r}^{x y}\right|$ $\rightarrow 0$ for better convergence. For calculating the correlation function $g\left(r^{x y}\right)$ on the $x-y$ plane, we have considered a disk (shown schematically in Fig. 1) which lies in the $x-y$ plane around each molecule, whose axis is along the $z$ direction and having a thickness of one molecular diameter. The distribution of molecules whose center of mass lies in the disk gives the planar distribution function around each molecule in the $x-y$ plane. Similarly for calculating the linear distri- 

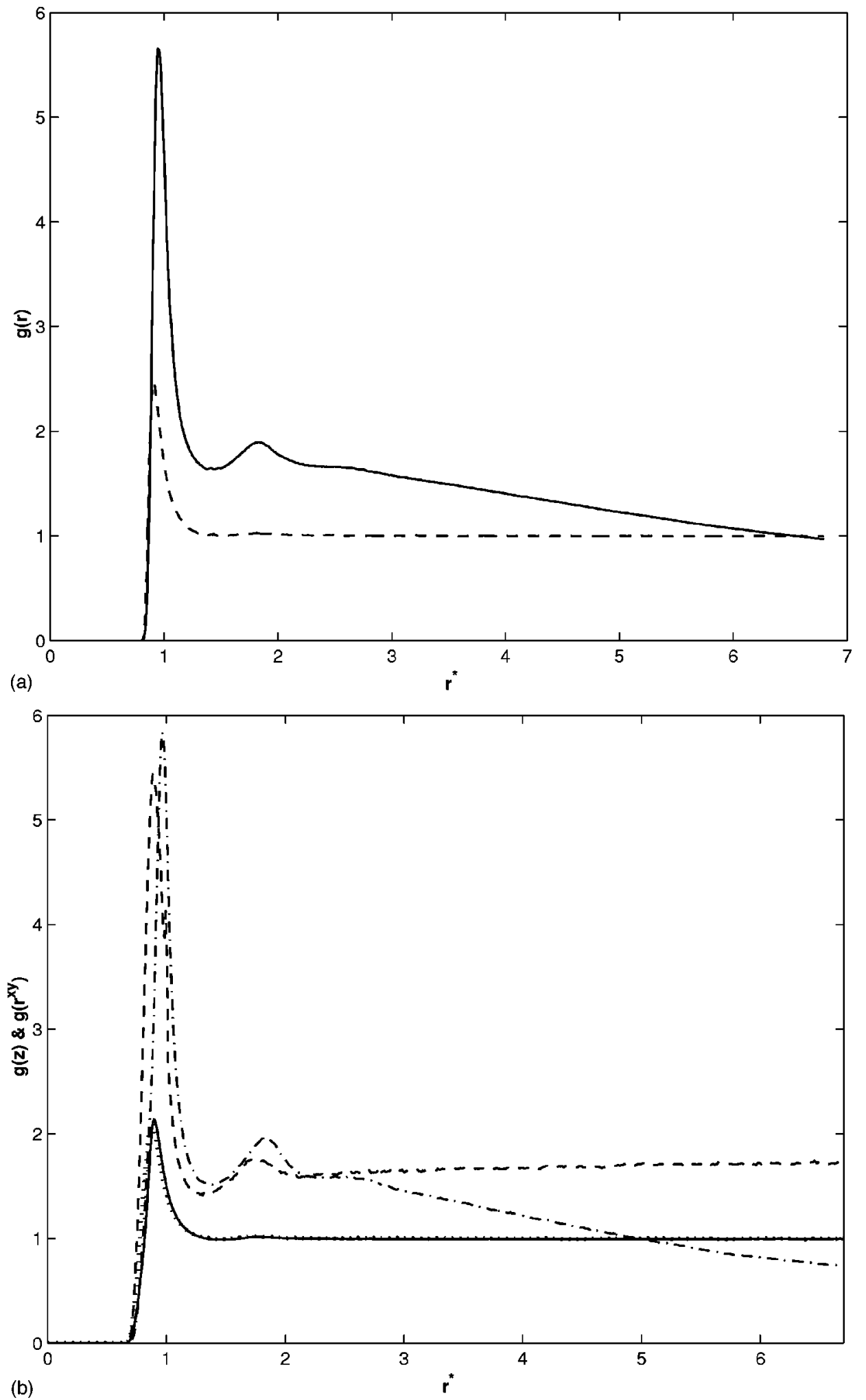

FIG. 2. (a) Radial distribution function of the solute molecules with an absence of radiation pressure is given. $g(r)$ without radiation pressure is given by dashed line, solid line gives the corresponding $g(r)$ with radiation pressure. Note that the solid line goes below 1.0 indicating the inhomogeneity in the distribution. (b) $g\left(r^{x y}\right)$ and the $g(z)$ are drawn to find the average density-density distribution in the $x$ $-y$ plane and in the $z$ direction, respectively. The dotted curve represents $g\left(r^{x y}\right)$ without perturbation. The dotted curve almost coincides with the bold line which is $g(z)$ without RP. When perturbation is applied the change in $g\left(r^{x y}\right)$ shown in the dotteddashed curve. The corresponding change in $g(z)$ is shown as dashed curve. Note that $g\left(r^{x y}\right)$ under perturbation goes below 1 at shorter distances indicating the formation of inhomogeneity in the $x-y$ plane. The shell structure formation in the $z$ direction under perturbation is indicated by the second peak in the dashed curve. bution function $g(z)$ we considered a cylinder whose diameter is that of one molecule with reference molecule inside this cylinder (shown schematically in Fig. 1). The densitydensity distribution function of molecules whose center of mass lies in this cylinder is calculated. These steps are repeated with perturbation to get the corresponding correlation functions. The distinct part of van Hove correlation function is defined as ${ }^{16}$

$$
G_{d}(\mathbf{r}, t)=\frac{1}{N}\left\langle\sum \sum_{i \neq j}^{N} \delta\left(\mathbf{r}+\mathbf{r}_{j}(0)-\mathbf{r}_{i}(t)\right)\right\rangle
$$

which gives the information about average lifetime of shell structure around each molecule. The van Hove correlation function in the unperturbed and the perturbed states are calculated for comparing the lifetime of shell structures in these 


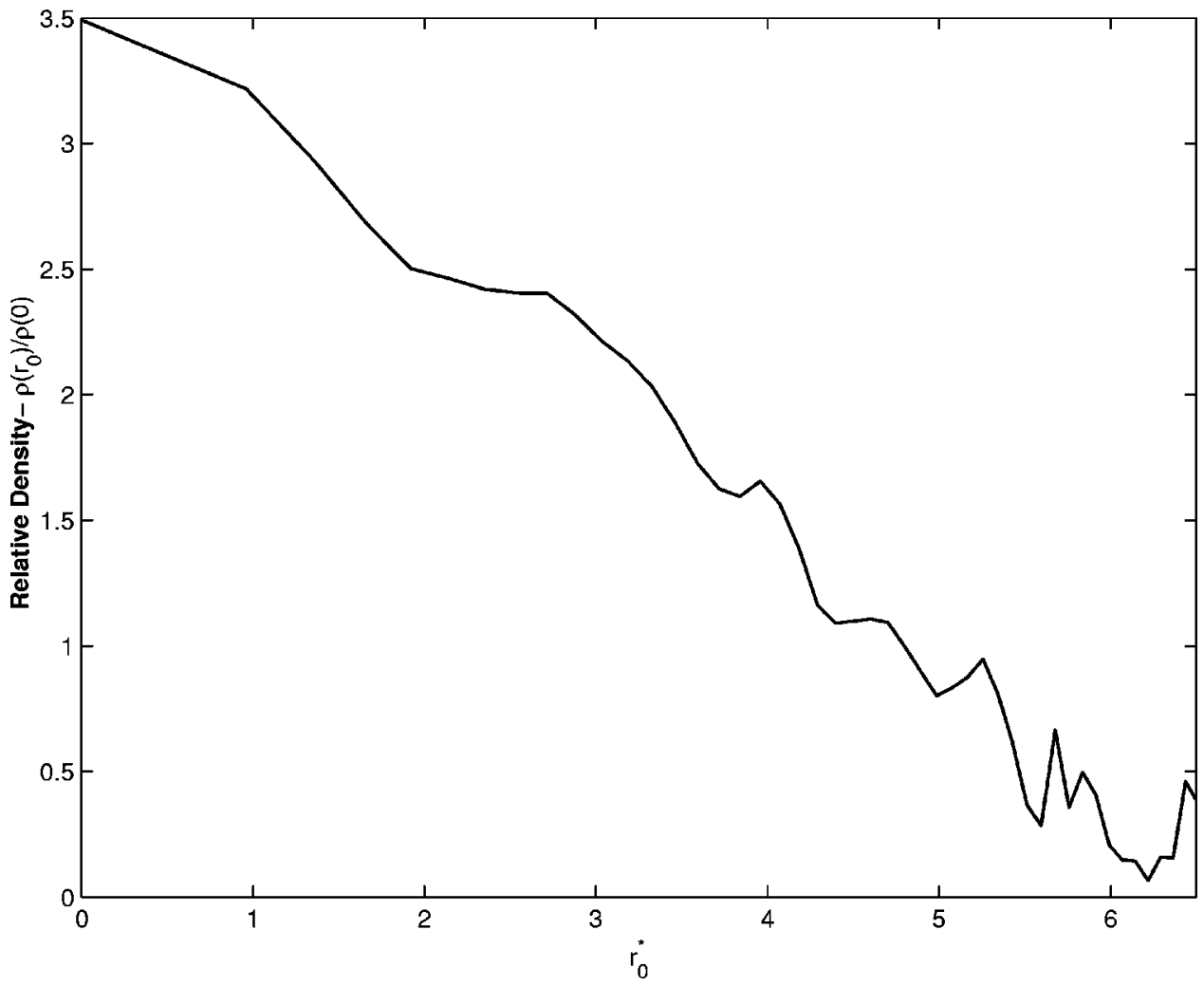

FIG. 3. Corresponding variation in the density plotted from an imaginary radiation axis (along the $z$-axis at the center of the $x-y$ plane) to the sides of the simulation box. In the presence of RP the density acquires an inhomogeneity. states. Since the perturbation is introduced by a position dependent external field the two body correlation function is not a reliable source of information about spatial distribution of molecules. Hence the density distribution of molecules in perturbed state is calculated from the stored positions which gives the spatial distribution of density.

For monitoring the variation of density with time, in the transformation from the nonequilibrium to an equilibrium state, immediately after the release of external perturbation, simulation starts from an equilibrated configuration with perturbation which is preserved till the end of the simulation. The simulation is performed for 10000 steps without perturbation and using the preserved positions as starting configuration. During this time, the density is monitored in the interval after each ten consecutive steps. Next initial configuration is generated by running the simulation for 1000 steps in the initial equilibrated configuration starting from the preserved positions. These simulation steps are repeated over 100 runs and the output data are averaged to obtain good statistics and to smooth the time evolution curve of density. In a similar way, transformation from unperturbed to perturbed state is also recorded.

\section{RESULTS AND DISCUSSION}

Figure 2(a) shows the $g(r)$ of 500 solute molecules. The dashed curve represents the $g(r)$ without any radiation and the solid curve gives the $g(r)$ in the presence of radiation pressure (RP). Change in the radial distribution function in Fig. 2(a) follows from the clustering of the solute molecules near the radiation axis. The $g(r)$ curve with perturbation shows a very high peak which is much higher than that of an ordinary solution. Since in the solution the solute molecules are in low density, the second peak of the $g(r)$ is not much visible in the absence of RP. But with the RP, the second peak becomes visible giving clear indication of the formation of second shell structure. Note that between the first and the second peak the density is much higher than the average density which shows the random structure of the clusters. $g\left(r^{x y}\right)$ and $g(z)$ are separately plotted in Fig. 2(b). In the absence of perturbation, these correlation functions show similar behavior allowing both curves to coincide one over the other. The dotted $\left[g\left(r^{x y}\right)\right]$ and the bold $[(g(z)]$ lines in Fig. 2 coincide almost one over the other, which is the signature of a homogeneous solution. Under the influence of the inhomogeneous perturbation due to the RP in the first peaks of the $g\left(r^{x y}\right)$ [dashed-dotted curve in Fig. 2(b)] and the $g(z)$ [dashed curve in Fig. 2(b)] rise to a very high value and their second peaks also become visible. This is an indication of explicit shell structure formation in the $x-y$ plane and in the $z$ direction, but their structures considerably differ from each other. This fact can be inferred from the difference in height between the first peak and the second peak positions of $g(z)$ and $g\left(r^{x y}\right)$ with the RP. Also the position of peaks of both curves in the perturbed state shifts from each other and their heights also differs giving different probability for position of nearest neighbors in different directions. The $g\left(r^{x y}\right)$ with RP goes below 1 at a distance of about 5 , giving an indication of high inhomogeneity in the distribution of molecules in the $x-y$ plane. $g(z)$ behaves similarly to that of the unperturbed case, but the peaks become more visible and this function decays to $g(z)=1.6$ instead of $g(z)=1$ at large distances, giving indication of the formation of a high density region along the radiation axis. This fact is also supported by variation of density from the radiation axis radially 

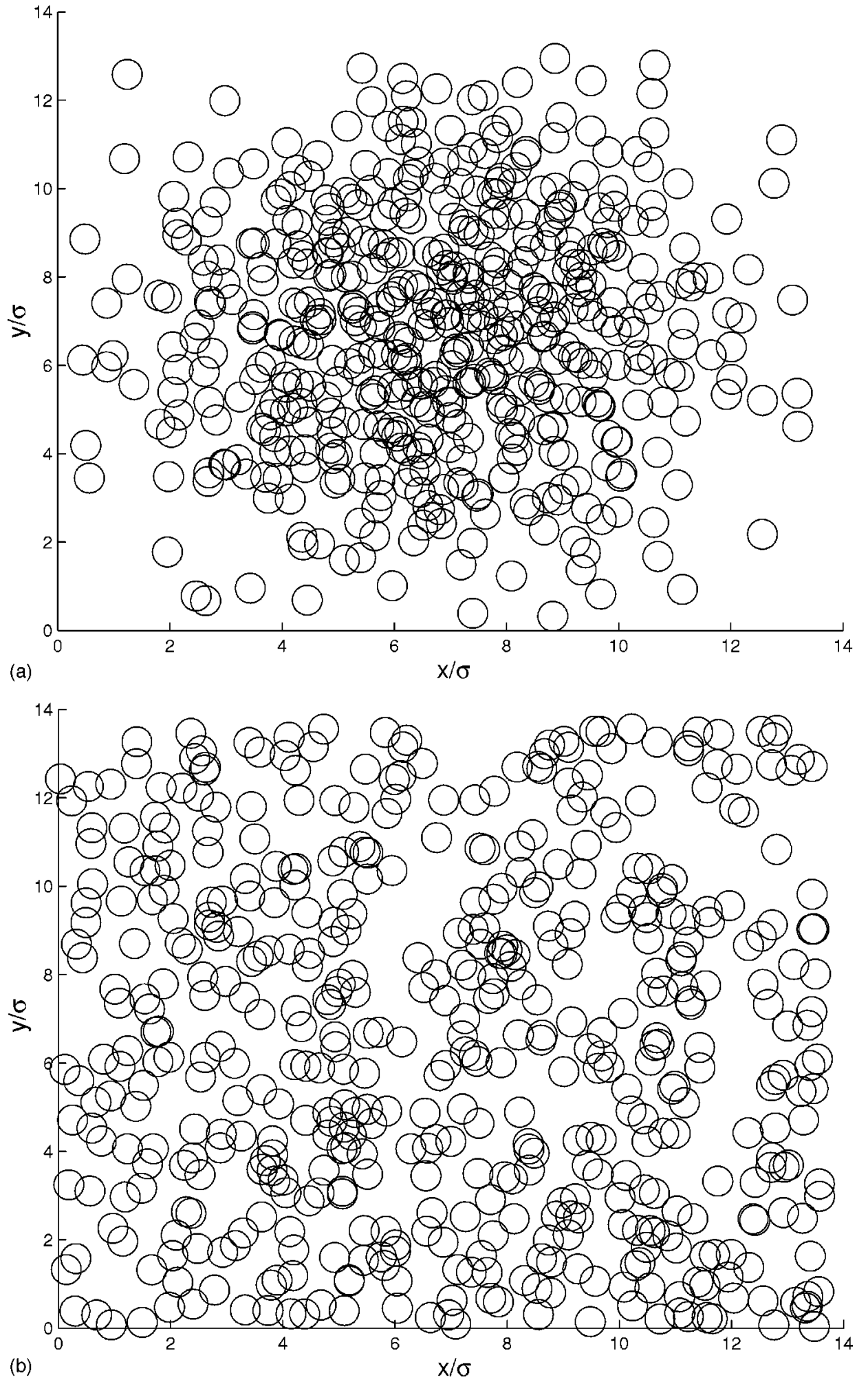

FIG. 4. Snapshot of molecules in the simulation box projected on the $x-y$ plane (a) with radiation pressure, (b) without radiation pressure. Circles are approximately the same size as molecule. (a) clearly indicates the clustering of molecules. outward as shown in Fig. 3; this gives the arrangement of clusters in the radiation field. The inhomogeneity in the force field is reflected in the rearrangement of density in the perturbed state. These clusters are visible in the snapshot of the molecules in the simulation box. Figure 4(a) gives the snapshot after equilibrium with perturbation. Here the clusters are at the center of the simulation box in comparison with the clusters in Fig. 4(b) which is the snapshot taken without perturbation.
Figure 5(a) gives the van Hove correlation function in an inhomogeneous solution; this gives the information about the collective dynamics of the molecules in the presence of the RP in equilibrium. In Fig. 5(a) $G_{d}(r, t)$ is plotted from time $0.5 t^{*}$ in the successive intervals of $0.5 t^{*}$. This gives information about how fast the local shell structure of liquid relaxes in the presence of the RP. Figure 5(b) gives the corresponding van Hove correlation function in the absence of RP. These curves are plotted from time $0.05 t^{*}$ in the successive 
intervals of $0.05 t^{*}$. The time interval used in Fig. 5(a) is ten times greater than that used in Fig. 5(a) is ten times greater than that used in Fig. 5(b). Hence the shell structure breaks down slowly in the perturbed state which essentially means the stability of the clusters in the presence of the RP. Figure $5(\mathrm{c})$ gives the plot of $C(t)$ which is defined as

$$
C(t)=\frac{G_{d}(r, t)-G_{d}(r, t=\infty)}{G_{d}(r, t=0)-G_{d}(r, t-\infty)}
$$

against time at $r$ equal to the peak position of first shell. The stars in Fig. 5(c) shows the behavior of $C(t)$ in the perturbed state and the circles gives the corresponding behavior in the unperturbed state. These curves give a quantitative comparison between the lifetime of clusters in perturbed and unperturbed solution, the relaxation time with RP is approximately ten times greater than that of without RP. The plot of density variation with time gives a clear picture of the dynamics of density fluctuations and the relaxation of solute molecules. The density (relative) variation with time at different points in the simulation box immediately after the application of the

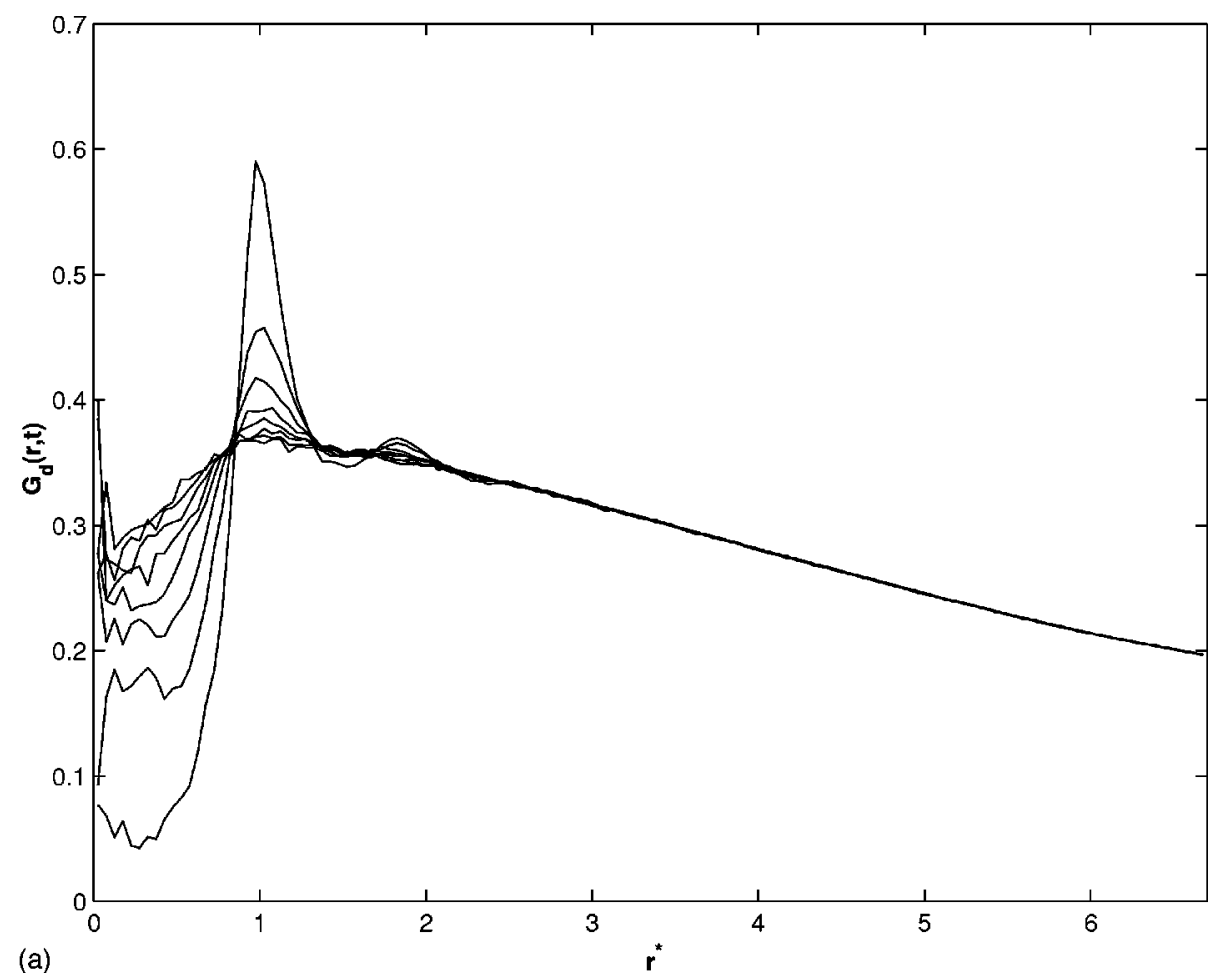

(a)

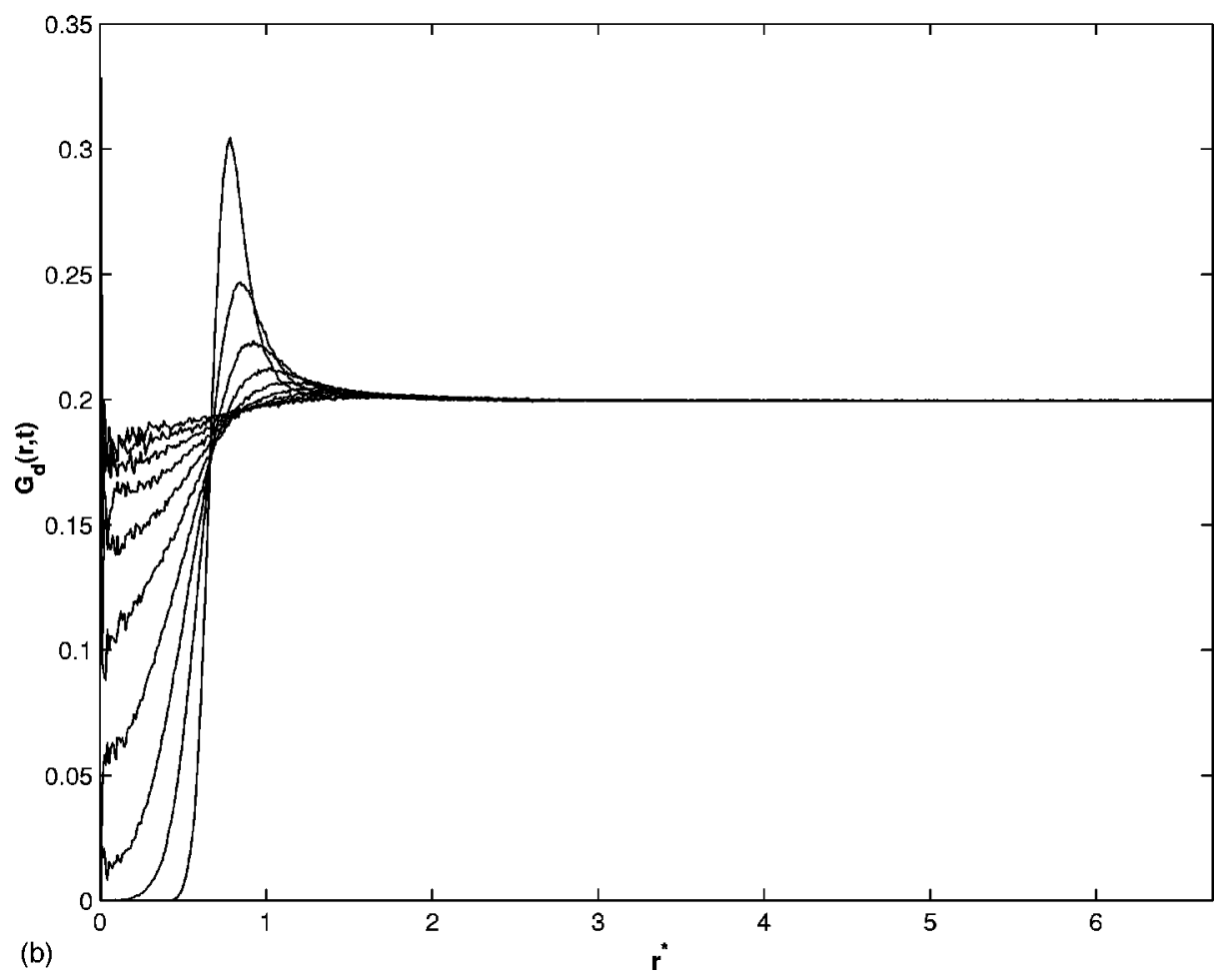

FIG. 5. Distinct part of the van Hove correlation function is calculated and plotted against the position in (a) the perturbed and (b) the unperturbed solutions. In (a) the successive curves starting near $r=0$ from bottom to top are separated by time interval of $0.5 t^{*}$ and the corresponding separation in (b) is $0.05 t^{*}$. The variation in time of the shell structures are plotted and compared in perturbed and unperturbed systems in (c). Here $C(t)$ is plotted against time at $r$ value equal to the peak position of the first shell. The circles give the $C(t)$ without RP and stars give $C(t)$ with RP. The shell structure breaks down slowly in the presence of RP which signifies the existence of relatively stabler clusters than in the unperturbed solution. 


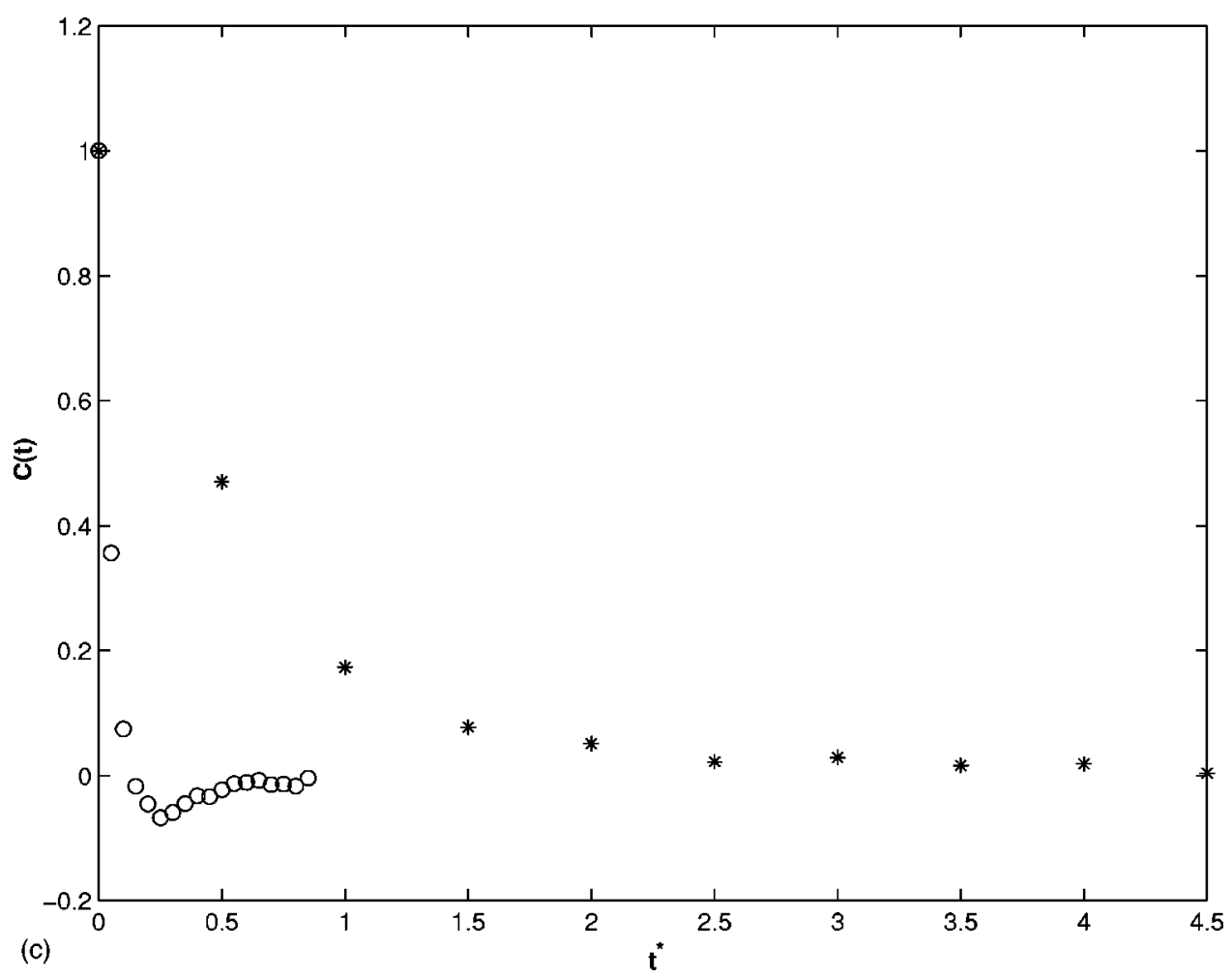

FIG. 5. (Continued.)

RP is plotted in Fig. 6. Density fluctuations at $r_{0}^{*}=0.0,1.7$, $2.5,3.0,3.5,4.0,4.3,4.6,5.0,5.3,5.5,5.8,6.0,6.3,6.6$ away from the radiation axis is the radial direction (of cylinder representing the beam) shows a similar kind of relaxation behavior and their relaxation time also does not appreciably differ. It is interesting to compare this relaxation behavior of the solute molecules in perturbed state with that experienced in the unperturbed state. Figure 7 shows the corresponding density variation in the system after the RP is removed. Here, due to the absence of RP, the solution is in a lower pressure state than the previous case. The density of solute is found to have a shorter relaxation time than the

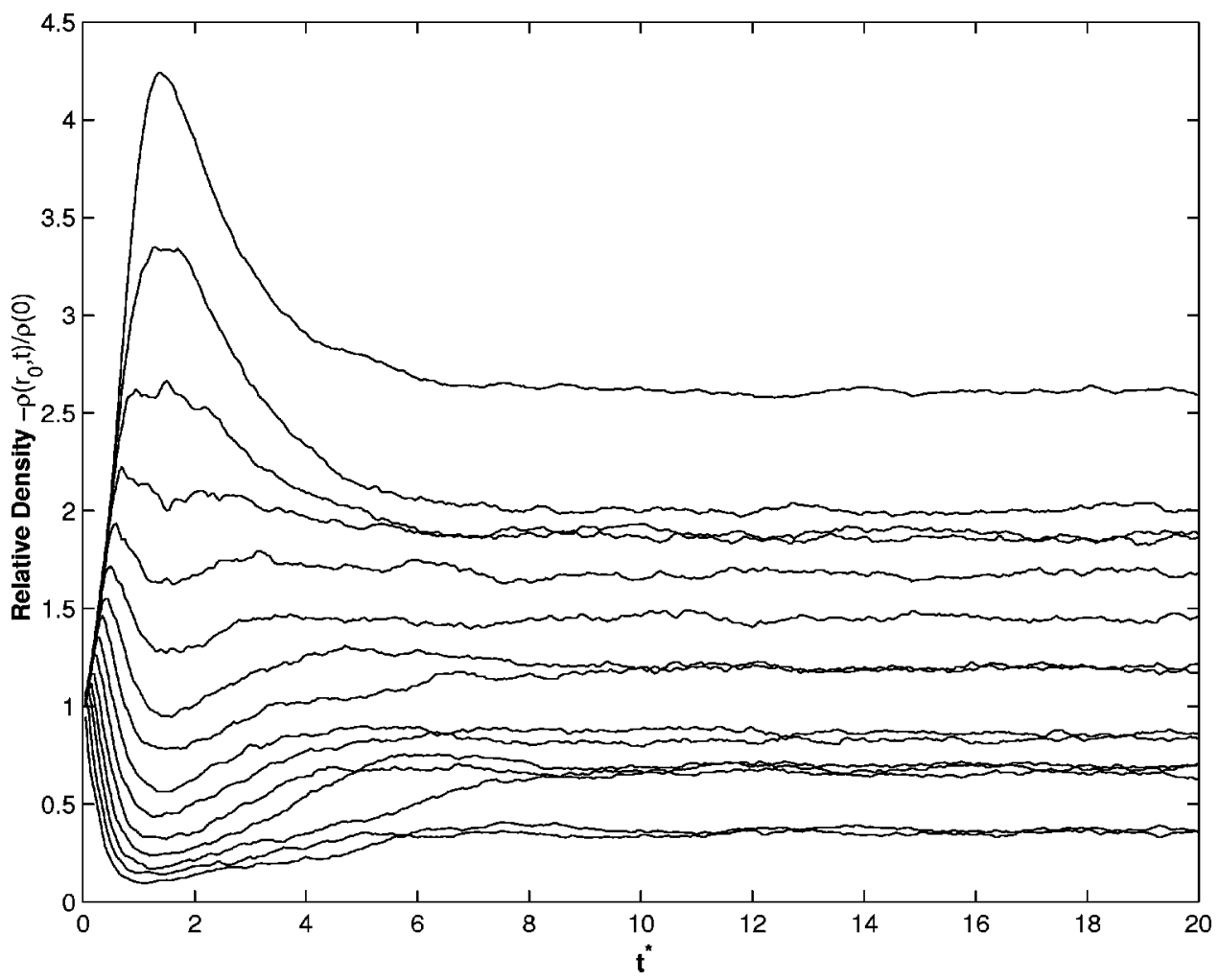

FIG. 6. Density variation at the center of the radiation axis with time after RP is applied. Different lines are plotted for representing the density variation at the different positions. These set of lines gives the density variation with time from the radiation axis at distances $0.0,1.7,2.5,3.0,3.5,4.0,4.3$, $4.6,5.0,5.3,5.5,5.8,6.0,6.3,6.6$ successively from top to bottom (near $t^{*}$ $=2$ ) away from the radiation axis. 


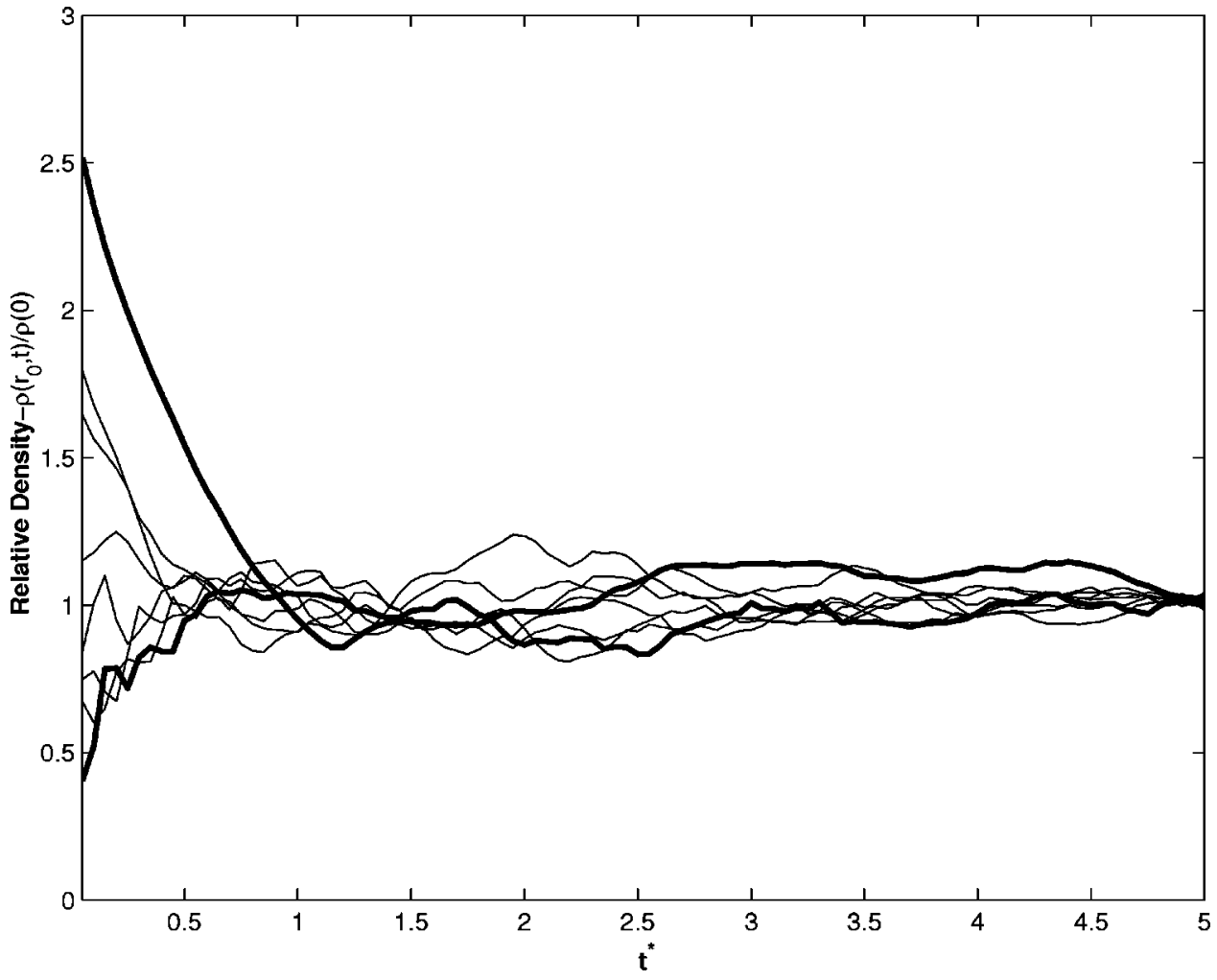

FIG. 7. Density variation at center of the radiation axis with time after RP is removed the different positions. These sets of lines give density variation with time from the radiation axis at distances $0.0,2.5,3.5,4.3,5.0,5.5$, $6.0,6.5$ successively from top to bottom (at $\left.t^{*}=0\right)$ away from the radiation axis. previous case. Here also density fluctuations at $r_{0}^{*}=0.0,2.5$, $3.5,4.3,5.0,5.5,6.0,6.5$ are plotted which show a similar kind of relaxation behavior.

\section{CONCLUSION}

In conclusion we have demonstrated the formation of nanoclusters under the radiation pressure. These clusters are found to be more stable than those formed in an unperturbed solution. We found that even though the force field resultingfrom the RP is confined to the $x-y$ plane, it modifies the shell structure in the $z$ direction also. The $g(z)$ is still found to be homogeneous but a strong inhomogeneity exists in the $g\left(r^{x y}\right)$. The van Hove correlation calculated in the unperturbed state will relax approximately ten times faster in the unperturbed state; this will substantiate the stability of clusters in the perturbed state. Moreover, we could observe the rich dynamical behavior during the formation and the dissolution of clusters and could observe the variation in the relaxation time in the perturbed and the unperturbed states.

It is interesting to note the oscillations in local density when the electromagnetic field is turned on or off. These oscillations are manifestations of the viscoelasticity of the liquid. If the linear response is valid, then one could possibly describe these oscillations by using the dynamic structure factor of the liquid. ${ }^{16}$ Such a calculation is nontrivial because the system becomes inhomogeneous in the presence of the position dependence of the field. Thus, one would require to use the density functional theory in real (that is, position) space. Such a calculation is computationally intensive, but may be worthwhile to perform.

\section{ACKNOWLEDGMENTS}

It is a pleasure to thank G. Srinivas for help and discussions. This work was supported in part in grants from the Department of Science and Technology, India and the Council of Scientific and Industrial Research, India.

${ }^{1}$ M. Lewerenz, B. Schilling, and J. P. Toennies, J. Chem. Phys. 102, 8191 (1995).

${ }^{2}$ H. Tanaka, J. Chem. Phys. 107, 3734 (1997).

${ }^{3}$ S. Jayanetti, R. A. Mayanovic, A. J. Anderson, W. A. Bassett, and I. M. Chou, J. Chem. Phys. 115, 954 (2001).

${ }^{4}$ P. Debye, Ann. Phys. (Leipzig) 30, 57 (1909).

${ }^{5} \mathrm{M}$. Kerker, The Scattering of Light and Other Electromagnetic Radiation (Academic, New York, 1969).

${ }^{6}$ J. P. Gordon, Phys. Rev. A 8, 14 (1973).

${ }^{7}$ A. Ashkin, Phys. Rev. Lett. 24, 156 (1970); A. Ashkin and J. M. Dziedzic, Appl. Phys. Lett. 19, 283 (1971).

${ }^{8}$ J. Hotta, K. Sasaki, and H. Masuhara, J. Am. Chem. Soc. 118, 11968 (1996); J. Hoffkens, J. Hotta, K. Sasaki, H. Masuhara, H. Faes, and F. C. DeSchryver. Mol. Cryst. Liq. Cryst. 283, 165 (1996).

${ }^{9}$ J. Hoffkens, J. Hotta, K. Sasaki, H. Masuhara, and K. Iwai, Langmuir 13, 414 (1997); J. Hoffkens, J. Hotta, K. Sasaki, H. Masuhara, T. Taniguchi, and T. Miyashita, J. Am. Chem. Soc. 119, 2741 (1997).

${ }^{10}$ K. Sasaki, M. Tsukima, and H. Masuhara, Appl. Phys. Lett. 71, 37 (1997); J. Won, T. Inaba, H. Masuhara, H. Fujiwara, K. Sasaki, S. Miyawaki, and S. Sato, ibid. 75, 1506 (1997).

${ }^{11}$ P. Borowicz, J. Hotta, K. Sasaki, and H. Masuhara, J. Phys. Chem. B 102, 1896 (1998); J. Hotta, K. Sasaki, H. Masuhara, and Y. Morishima, ibid. 102, 7687 (1998); T. A. Smith, J. Hotta, K. Sasaki, H. Masuhara, and Y. Itoh, ibid. 103, 1660 (1999).

${ }^{12}$ Y. R. Shen, The Principles of Nonlinear Optics (Wiley, New York, 1984).

${ }^{13}$ A. K. Ghatak and K. Thyagarajan, Optical Electronics (Cambridge University Press, Cambridge, 1991).

${ }^{14}$ M. P. Allen and D. J. Tildesley, Computer Simulation of Liquids (Clarendon Press, Oxford, 1987).

${ }^{15}$ D. L. Ermak and J. A. McCammon, J. Chem. Phys. 69, 1352 (1978).

${ }^{16}$ J. P. Hansen and I. R. McDonald, Theory of Simple Liquids (Academic, New York, 1976). 